\title{
Bright and singular soliton solutions of Tzitzéica type equations appear in nonlinear optics
}

\author{
Asim Zafar ${ }^{1, *}$, Alper Korkmaz ${ }^{2}$, Muhammad Imran Qureshi ${ }^{1}$ and Asfand Fahad ${ }^{1}$ \\ ${ }^{1}$ Department of Mathematics, COMSATS University Islamabad, Vehari Campus, Pakistan. \\ ${ }^{2}$ Department of Mathematics, Çankırı Karatekin University, Çankırı, Turkey.
}

\begin{abstract}
We investigate the exact travelling wave solutions of the Tzitzéica-Dodd-Bullough-Mikhailov (TDBM) and Tzitzéica-type (TT) equations, appear in nonlinear optics, using simple ansatz approach. We use the Painlevé and traveling wave transformations to convert the mentioned nonlinear equations into nonlinear ordinary differential equations. Then, the simple ansatz approach has been employed to procure wave solutions, in terms of bright, singular soliton and trigonometric functions, for the TzitzéicaDodd-Bullough-Mikhailov (TDBM) and Tzitzéica-type (TT) equations. The study shows that the simple ansatz approach is straightforward and easy to implement for solving nonlinear problems.
\end{abstract}

Keywords: Tzitzéica-Dodd-Bullough-Mikhailov equation, Tzitzéica-type equation, ansatz approach, traveling wave solution, periodic solutions.

\section{Introduction}

Evolution equations with nonlinear terms that appear in optics consist of the TzitzéicaDodd-Bullough-Mikhailov(TDBM), Tzitzéica-Dodd-Bullough(TDB), and the Liouville equations. The nonlinearities in the equations in this class include exponential functions. Consider the Tzitzéica-Dodd-Bullough-Mikhailov equation

$$
u_{x t}+e^{u}+e^{-2 u}=0
$$

firstly defined in Tzitzéica's study [1] in 1908. In the following years a variational form of the (1) was used in various studies as in $[2,3]$

$$
u_{t t}+u_{x x}+e^{u}+e^{-2 u}=0
$$

${ }^{*}$ Corresponding author: asimzafar@hotmail.com 
named Tzitzéica-type(TT) equation. The conditions of the existence of polynomial conserved densities for the TDBM equation (1) in terms of derivatives of $u$ were listed in [4]. Bäcklund transformation for the TDBM equation from the classification of PainleveGambier was given in details by Conte et al [5]. Zhu and Geng presented Darboux transformation for the $3 \times 3$-matrix spectral TDBM equation problem and derived some explicit solutions [6]. The inverse problem was solved and $N$-soliton solutions for the TDBM were found besides infinitely many conservation laws were defined for the Toda chain system equations in two dimensions [7]. One should note that Mikailov [7] claimed Conte et al. [5]'s suggestion on the polynomial conserved densities was not true. Some logarithmic and exponential function type solutions to the TDBM (1) were explicitly constructed by some Fourier series approach by El-Kalaawy [8]. The modification of the simple equation approach was also used to set the traveling wave solutions in terms of logarithmic functions with hyperbolic or trigonometric parameters to the TDBM (1) by employing two variable transformations to it [9]. Blow-up and broken singular traveling wave solutions covering periodic, solitary wave, kink and unbounded wave forms were successfully given with their plots for both the TDBM (1) and the TT (2) equations by integral bifurcation approach [2]. Soliton and periodic solutions were derived by the hyperbolic tangent method together with the Painleve property to both the TDBM (1) and the TT (2) equations [3].

Parallel developments in both computer technologies and symbolic softwares have greatly contributed to solve lots of problems defined in various fields covering applied mathematics, physics and many engineering fields. A diverse class of effective methods have successfully been introduced to study this class of equations, for example [3,10-15]. On the other hand some of the commonly used approaches, for solving nonlinear evolution equations, are: The ansatz [16-18], modified simple equation [19], the first integral [20,21], $\left(\frac{G^{\prime}}{G}\right)$-expansion [22], sine-Gordon expansion [23,24]. Furthermore, some other excellent works like Kudryashov methods [25], a modified form of Kudryashov and functional variable methods [26-28] have been done by different researchers. In [29-32], the auxiliary equation, the improved $\tan \left(\frac{\phi(\eta)}{2}\right)$-expansion methods and the exp function approach have been explored for discrete and fractional order PDEs as well. Ali and Hassan [33], Hosseini et al. [34] and Zayed and Al-Nowehy [35] all have utilized the $\exp _{a}$ function method to explore the exact solutions of nonlinear partial differential equations. A useful study of hyperbolic function approach has been given in [36-38].

The investigation for exact solutions is as follows. The description of simple ansatz approach is provided in section 2. The method is utilized to extract new exact solutions of nonlinear Tzitzéica type equations in section 3. The conclusion is provided in the last section. 


\section{A simple ansatz approach}

The present section provides a succinct description of the simple ansatz approach [39]. For this purpose, suppose that a nonlinear PDE can be read as

$$
P\left(u, \frac{\partial u}{\partial x}, \frac{\partial u}{\partial t}, \frac{\partial^{2} u}{\partial x^{2}}, \frac{\partial^{2} u}{\partial x \partial t}, \frac{\partial u}{\partial t^{2}}, \ldots\right)=0
$$

The PDE (3) can be changed into the following ODE

$$
Q\left(U, U^{\prime}, U^{\prime \prime}, \ldots\right)=0
$$

by the classical wave transform given below

$$
u(x, t)=U(\xi), \xi=k x+l t,
$$

in which $k, l$ are non-zero constants. Then, we assume the solution of the form given by,

$$
U(\xi)=\alpha_{0}+\sum_{i=1}^{N} \alpha_{i} \psi^{i}(\xi)
$$

where, $\alpha_{0}, \alpha_{1}, \alpha_{2}, \ldots, \alpha_{N}$ are constant parameters and $N$ is positive natural number that will be found by the principle of homogeneous balance. $\psi(\xi)$ is deliberately chosen to be $\operatorname{csch}(\xi)$ and $\operatorname{sech}(\xi)$ to determine hyperbolic type solution while $\sec (\xi)$ and $\csc (\xi)$ are periodic solutions, respectively.

\subsection{Solutions for the TDBM equation}

The DTDBM equation is read as

$$
u_{x t}+e^{u}+e^{-2 u}=0
$$

and using Painlevé transform $v=e^{u}$ or $u=\ln (v)$, the Eq. (7) reduces to:

$$
v v_{x t}-v_{x} v_{t}+v^{3}+1=0
$$

Eq.(8) is reduced to the following ODE by using $v(x, t)=V(\xi), \xi=k x+l t$ :

$$
k l\left(V V^{\prime \prime}-V^{\prime 2}\right)+V^{3}+1=0
$$

The balance between the terms $V V^{\prime \prime}$ with $V^{3}$ implies $N=2$.

$$
V(\xi)=\alpha_{0}+\alpha_{1} \operatorname{csch}(\xi)+\alpha_{2} \operatorname{csch}^{2}(\xi)
$$


By inserting Eq.(10) with its derivatives in Eq.(9) and setting each coefficient of powers of $\operatorname{csch}(\xi)$ to zero, one obtains a nonlinear system of algebraic equations.

The solution of this system gives

$$
\begin{aligned}
& \alpha_{0}=-1, \alpha_{1}=0, \alpha_{2}=-\frac{3}{2}, k=k, l=\frac{3}{4 k} \\
& \alpha_{0}=\frac{1}{2}-i \frac{\sqrt{3}}{2}, \alpha_{1}=0, \alpha_{2}=\frac{3}{4}-i \frac{3 \sqrt{3}}{4}, k=k, l=\frac{3 i(\sqrt{3}+i)}{8 k} \\
& \alpha_{0}=\frac{1}{2}+i \frac{\sqrt{3}}{2}, \alpha_{1}=0, \alpha_{2}=\frac{3}{4}+i \frac{3 \sqrt{3}}{4}, k=k, l=-\frac{3 i(\sqrt{3}-i)}{8 k}
\end{aligned}
$$

We have the following hyperbolic function solutions

$$
\begin{aligned}
& u_{1}(x, t)=\ln \left(-\frac{3}{2} \operatorname{csch}^{2}\left(k x+\frac{3}{4 k} t\right)-1\right) \\
& u_{2}(x, t)=\ln \left(\frac{1}{2}-i \frac{\sqrt{3}}{2}+\left(\frac{3}{4}-i \frac{3 \sqrt{3}}{4}\right) \operatorname{csch}^{2}\left(k x+\frac{3 i(\sqrt{3}+i)}{8 k} t\right)\right) \\
& u_{3}(x, t)=\ln \left(\frac{1}{2}+i \frac{\sqrt{3}}{2}+\frac{3}{4}+i\left(\frac{3 \sqrt{3}}{4}\right) \operatorname{csch}^{2}\left(k x-\frac{3 i(\sqrt{3}-i)}{8 k} t\right)\right)
\end{aligned}
$$

For more hyperbolic function solution, we take $\psi(\xi)=\operatorname{sech}(\xi)$

$$
V(\xi)=\alpha_{0}+\alpha_{1} \operatorname{sech}(\xi)+\alpha_{2} \operatorname{sech}^{2}(\xi) ; \xi=k x+l t
$$

By inserting Eq.(14) into Eq.(9), and gather the terms with the same powers of $\operatorname{sech}(\xi)$. Then, by putting each coefficient of $\operatorname{sech}(\xi)$ equal to zero, we get a set of nonlinear algebraic equations. On solving, we procure the following set of solutions

$$
\begin{aligned}
& \alpha_{0}=-1, \alpha_{1}=0, \alpha_{2}=\frac{3}{2}, l=l, k=\frac{3}{4 l} \\
& \alpha_{0}=\frac{1}{2}+i \frac{\sqrt{3}}{2}, \alpha_{1}=0, \alpha_{2}=\frac{-3}{4}-i \frac{3 \sqrt{3}}{4}, l=l, k=-\frac{3 i(\sqrt{3}-i)}{8 l} \\
& \alpha_{0}=\frac{1}{2}-i \frac{\sqrt{3}}{2}, \alpha_{1}=0, \alpha_{2}=\frac{-3}{4}+i \frac{3 \sqrt{3}}{4}, l=l, k=\frac{3 i(\sqrt{3}+i)}{8 l}
\end{aligned}
$$

Thus, substitution of the above values into the Eq. (7), the solutions are set as follows:

$$
\begin{aligned}
& u(x, t)=\ln \left(-1+\frac{3}{2} \operatorname{sech}^{2}\left(\frac{3}{4 l} x+l t\right)\right) \\
& u(x, t)=\ln \left(\frac{1}{2}+i \frac{\sqrt{3}}{2}+\left(\frac{-3}{4}-i \frac{3 \sqrt{3}}{4}\right) \operatorname{sech}^{2}\left(-\frac{3 i(\sqrt{3}-i)}{8 l} x+l t\right)\right) \\
& u(x, t)=\ln \left(\frac{1}{2}-i \frac{\sqrt{3}}{2}+\left(\frac{-3}{4}+i \frac{\sqrt{3}}{4}\right) \operatorname{sech}^{2}\left(\frac{3 i(\sqrt{3}+i)}{8 l} x+l t\right)\right)
\end{aligned}
$$




\subsection{Periodic solutions for the TDBM equation}

For periodic solutions, we start as follows:

$$
V(\xi)=\alpha_{0}+\alpha_{1} \sec (\xi)+\alpha_{2} \sec ^{2}(\xi)
$$

Setting coefficients of power of $\sec (\xi)$ to zero yields an algebraic system of equations. Thus, the solution gives

$$
\begin{aligned}
& \alpha_{0}=-1, a_{1}=0, a_{2}=\frac{3}{2}, k=k, l=-\frac{3}{4 k} \\
& \alpha_{0}=\frac{1}{2}+i \frac{\sqrt{3}}{2}, \alpha_{1}=0, \alpha_{2}=\frac{-3}{4}-i \frac{\sqrt{3}}{4}, k=k, l=\frac{3(1+i \sqrt{3})}{8 k} \\
& \alpha_{0}=\frac{1}{2}-i \frac{\sqrt{3}}{2}, \alpha_{1}=0, \alpha_{2}=\frac{-3}{4}+i \frac{\sqrt{3}}{4}, k=k, l=\frac{3(1-i \sqrt{3})}{8 k}
\end{aligned}
$$

the following trigonometric function solutions

$$
\begin{aligned}
& u_{1}(x, t)=\ln \left(\frac{3}{2} \sec ^{2}\left(k x-\frac{3}{4 k} t\right)-1\right) \\
& u_{2}(x, t)=\ln \left(\frac{1}{2}+i \frac{\sqrt{3}}{2}+\left(\frac{-3}{4}-i \frac{3 \sqrt{3}}{4}\right) \sec ^{2}\left(k x+\frac{3(1+i \sqrt{3})}{8 k} t\right)\right) \\
& u_{3}(x, t)=\ln \left(\frac{1}{2}-i \frac{\sqrt{3}}{2}+\left(\frac{-3}{4}+i \frac{\sqrt{3}}{4}\right) \sec ^{2}\left(k x+\frac{3(1-i \sqrt{3})}{8 k} t\right)\right)
\end{aligned}
$$

For more periodic solutions, we take the following non-trivial solution:

$$
V(\xi)=\alpha_{0}+\alpha_{1} \csc (\xi)+\alpha_{2} \csc ^{2}(\xi)
$$

Inserting Eq.(22) and its derivatives into Eq.(9) gives

$$
\begin{aligned}
& \alpha_{0}=-1, \alpha_{1}=0, \alpha_{2}=\frac{3}{2}, k=k, l=-\frac{3}{4 k} \\
& \alpha_{0}=\frac{1}{2}+i \frac{\sqrt{3}}{2}, \alpha_{1}=0, \alpha_{2}=\frac{-3}{4}-i \frac{\sqrt{3}}{4}, k=k, l=\frac{3(1+i \sqrt{3})}{8 k} \\
& \alpha_{0}=\frac{1}{2}-i \frac{\sqrt{3}}{2}, \alpha_{1}=0, \alpha_{2}=\frac{-3}{4}+i \frac{\sqrt{3}}{4}, k=k, l=\frac{3(1-i \sqrt{3})}{8 k}
\end{aligned}
$$

Thus the following trigonometric function solutions are obtained.

$$
\begin{aligned}
& u_{1}(x, t)=\ln \left(\frac{3}{2} \csc ^{2}\left(k x-\frac{3}{4 k} t\right)-1\right) \\
& u_{2}(x, t)=\ln \left(\frac{1}{2}+i \frac{\sqrt{3}}{2}+\left(\frac{-3}{4}-i \frac{\sqrt{3}}{4}\right) \csc ^{2}\left(k x+\frac{3(1+i \sqrt{3})}{8 k} t\right)\right) \\
& u_{3}(x, t)=\ln \left(\frac{1}{2}-i \frac{\sqrt{3}}{2}+\left(\frac{-3}{4}+i \frac{\sqrt{3}}{4}\right) \csc ^{2}\left(k x+\frac{3(1-i \sqrt{3})}{8 k} t\right)\right)
\end{aligned}
$$




\subsection{Solutions for the TT equation}

The TT equation is read as

$$
u_{t t}-u_{x x}-e^{u}+e^{-2 u}=0
$$

using the Painlevé transformation $v=e^{u}$ or $u=\ln (v)$, the Eq.(26) reduces to

$$
v v_{t t}-v v_{x x}-v_{t}^{2}+v_{x}^{2}-v^{3}+1=0
$$

Let us introduce a transformation $v(x, t)=V(\xi), \xi=k x+l t$, Eq.(27) can be turned into an ODE:

$$
\left(l^{2}-k^{2}\right)\left(V V^{\prime \prime}-V^{\prime 2}\right)-V^{3}+1=0
$$

Through balancing principle, we get $N=2$, then the non-trivial solution can be read as

$$
V(\xi)=\alpha_{0}+\alpha_{1} \operatorname{csch}(\xi)+\alpha_{2} \operatorname{csch}^{2}(\xi)
$$

by inserting Eq.(29) into Eq.(28), and rearranging the terms with the same power of $\operatorname{csch}(\xi)$ together.

Then, putting each coefficient of $\operatorname{csch}(\xi)$ equal to zero, we obtain a set of nonlinear algebraic equations. On solving, we find the following set of solutions

$$
\begin{aligned}
& \alpha_{0}=-\frac{1}{2}-i \frac{\sqrt{3}}{2}, \alpha_{1}=0, \alpha_{2}=-\frac{3}{4}-i \frac{3 \sqrt{3}}{4}, k=k, l=\mp \frac{\sqrt{2 k^{2}-\frac{3 i \sqrt{3}}{4}-\frac{3}{4}}}{\sqrt{2}} \\
& \alpha_{0}=-\frac{1}{2}+i \frac{\sqrt{3}}{2}, \alpha_{1}=0, \alpha_{2}=-\frac{3}{4}+i \frac{3 \sqrt{3}}{4}, k=k, l=\mp \frac{\sqrt{2 k^{2}+\frac{3 i \sqrt{3}}{4}-\frac{3}{4}}}{\sqrt{2}} \\
& \alpha_{0}=1, \alpha_{1}=0, \alpha_{2}=\frac{3}{2}, k=k, l=\mp \frac{1}{2} \sqrt{4 k^{2}+3}
\end{aligned}
$$

Thus, by substituting the above values into Eq. (29), the following new exact solutions can be written as

$$
\begin{aligned}
& u_{1,2}(x, t)=\ln \left(-\frac{1}{2}-i \frac{\sqrt{3}}{2}+\left(\frac{-3}{4}-i \frac{3 \sqrt{3}}{3}\right) \operatorname{csch}^{2}\left(k x \mp \frac{\sqrt{2 k^{2}-\frac{3 i \sqrt{3}}{4}-\frac{3}{4}}}{\sqrt{2}} t\right)\right) \\
& u_{3,4}(x, t)=\ln \left(-\frac{1}{2}+i \frac{\sqrt{3}}{2}+\left(\frac{-3}{4}+i \frac{3 \sqrt{3}}{3}\right) \operatorname{csch}^{2}\left(k x \mp \frac{\sqrt{2 k^{2}+\frac{3 i \sqrt{3}}{4}-\frac{3}{4}}}{\sqrt{2}} t\right)\right) \\
& u_{5,6}(x, t)=\ln \left(\frac{3}{2} \operatorname{csch}^{2}\left(k x \mp \frac{1}{2} \sqrt{4 k^{2}+3} t\right)+1\right)
\end{aligned}
$$

For more hyperbolic function solution, we take the non trivial solution as

$$
V(\xi)=\alpha_{0}+\alpha_{1} \operatorname{sech}(\xi)+\alpha_{2} \operatorname{sech}^{2}(\xi) ; \xi=k x+l t
$$


Substituting Eq.(33) into Eq.(28), and rearranging the terms with the same power of $\operatorname{sech}(\xi)$ together.

Then, by putting each coefficient of $\operatorname{sech}(\xi)$ equal to zero, we approach to a set of nonlinear algebraic equations. On solving, we find the following set of solutions

$$
\begin{aligned}
& \alpha_{0}=\frac{-1}{2}+i \frac{\sqrt{3}}{2}, \alpha_{1}=0, \alpha_{2}=\frac{3}{4}-i \frac{3 \sqrt{3}}{4}, k=k, l=\mp \frac{\sqrt{2 k^{2}+\frac{3 i \sqrt{3}}{4}-\frac{3}{4}}}{\sqrt{2}} \\
& \alpha_{0}=\frac{-1}{2}-i \frac{\sqrt{3}}{2}, \alpha_{1}=0, \alpha_{2}=\frac{3}{4}+i \frac{3 \sqrt{3}}{4}, k=k, l=\mp \frac{\sqrt{2 k^{2}-\frac{3 i \sqrt{3}}{4}-\frac{3}{4}}}{\sqrt{2}} \\
& \alpha_{0}=1, \alpha_{1}=0, \alpha_{2}=-\frac{3}{2}, l=\mp \frac{1}{2} \sqrt{4 k^{2}+3}
\end{aligned}
$$

Thus, by substituting the above values into Eq.(33), the new exact solutions can be written as follows:

$$
\begin{aligned}
& u_{1,2}(x, t)=\ln \left(\frac{-1}{2}+i \frac{\sqrt{3}}{2}+\left(\frac{3}{4}-i \frac{3 \sqrt{3}}{4}\right) \operatorname{sech}^{2}\left(k x \mp \frac{\sqrt{2 k^{2}+\frac{3 i \sqrt{3}}{4}-\frac{3}{4}}}{\sqrt{2}} t\right)\right) \\
& u_{3,4}(x, t)=\ln \left(\frac{-1}{2}-i \frac{\sqrt{3}}{2}+\left(\frac{3}{4}+i \frac{3 \sqrt{3}}{4}\right) \operatorname{sech}^{2}\left(k x \mp \frac{\sqrt{2 k^{2}-\frac{3 i \sqrt{3}}{4}-\frac{3}{4}}}{\sqrt{2}} t\right)\right) \\
& u_{5,6}(x, t)=\ln \left(\left|-\frac{3}{2} \operatorname{sech}^{2}\left(k x \mp \frac{1}{2} \sqrt{4 k^{2}+3} t\right)+1\right|\right)
\end{aligned}
$$

\subsection{Periodic solutions for TT equation}

We start with the following solution for Eq. (26).

$$
V(\xi)=\alpha_{0}+\alpha_{1} \sec (\xi)+\alpha_{2} \sec ^{2}(\xi) ; \xi=k x+l t
$$

Substituting Eq.(37) into Eq.(28), and collecting all terms with the same power of $\sec (\xi)$ together.

Then, by setting each coefficient of $\sec (\xi)$ equal to zero, we obtain a set of nonlinear algebraic equations. On solving, we find the following set of solutions:

$$
\begin{aligned}
& \alpha_{0}=\frac{-1}{2}+i \frac{\sqrt{3}}{2}, \alpha_{1}=0, \alpha_{2}=\frac{3}{4}-i \frac{3 \sqrt{3}}{4}, l=\mp \frac{\sqrt{2 k^{2}-\frac{3 i \sqrt{3}}{4}+\frac{3}{4}}}{\sqrt{2}} \\
& \alpha_{0}=\frac{-1}{2}-i \frac{\sqrt{3}}{2}, \alpha_{1}=0, \alpha_{2}=\frac{3}{4}+i \frac{3 \sqrt{3}}{4}, l=\mp \frac{\sqrt{2 k^{2}+\frac{3 i \sqrt{3}}{4}+\frac{3}{4}}}{\sqrt{2}} \\
& \alpha_{0}=1, \alpha_{1}=0, \alpha_{2}=-\frac{3}{2}, l=\mp \frac{1}{2} \sqrt{4 k^{2}-3}
\end{aligned}
$$


Thus, by substituting the above values into the Eq.(37), the new periodic solutions can be derived as follows:

$$
\begin{aligned}
& u_{1,2}(x, t)=\ln \left(\frac{-1}{2}+i \frac{\sqrt{3}}{2}+\left(\frac{3}{4}-i \frac{3 \sqrt{3}}{4}\right) \sec ^{2}\left(k x \mp \frac{\sqrt{2 k^{2}-\frac{3 i \sqrt{3}}{4}+\frac{3}{4}}}{\sqrt{2}} t\right)\right) \\
& u_{3,4}(x, t)=\ln \left(\frac{-1}{2}-i \frac{\sqrt{3}}{2}+\left(\frac{3}{4}+i \frac{3 \sqrt{3}}{4}\right) \sec ^{2}\left(k x \mp \frac{\left.\left.\sqrt{2 k^{2}+\frac{3 i \sqrt{3}}{4}+\frac{3}{4}} t\right)\right)}{\sqrt{2}}\right.\right. \\
& u_{5,6}(x, t)=\ln \left(-\frac{3}{2} \sec ^{2}(k x+l t) \mp \frac{1}{2} \sqrt{4 k^{2}-3}\right)
\end{aligned}
$$

Again for periodic solutions in terms of $\csc (\xi)$, we have

$$
V(\xi)=\alpha_{0}+\alpha_{1} \csc (\xi)+\alpha_{2} \csc ^{2}(\xi) ; \xi=k x+l t
$$

Substituting Eq.(41) into Eq.(28), and rearranging the terms with the same power of $\csc (\xi)$ together.

Then, by setting each coefficient of $\csc (\xi)$ equal to zero, we obtain a set of nonlinear algebraic equations. On solving, we procure the following set of solutions:

$$
\begin{aligned}
& \alpha_{0}=\frac{-1}{2}+i \frac{\sqrt{3}}{2}, \alpha_{1}=0, \alpha_{2}=\frac{3}{4}-i \frac{3 \sqrt{3}}{4}, k=k, l=\mp \frac{\sqrt{2 k^{2}-\frac{3 i \sqrt{3}}{4}+\frac{3}{4}}}{\sqrt{2}} \\
& \alpha_{0}=\frac{-1}{2}-i \frac{\sqrt{3}}{2}, \alpha_{1}=0, \alpha_{2}=\frac{3}{4}+i \frac{3 \sqrt{3}}{4}, k=k, l=\mp \frac{\sqrt{2 k^{2}+\frac{3 i \sqrt{3}}{4}+\frac{3}{4}}}{\sqrt{2}} \\
& \alpha_{0}=1, \alpha_{1}=0, \alpha_{2}=-\frac{3}{2}, k=k, l=\mp \frac{1}{2} \sqrt{4 k^{2}-3}
\end{aligned}
$$

Thus, by substituting the above values into the Eq.(41), the new periodic solutions can be written as follows:

$$
\begin{aligned}
& u_{1,2}(x, t)=\ln \left(\frac{-1}{2}+i \frac{\sqrt{3}}{2}+\left(\frac{3}{4}-i \frac{3 \sqrt{3}}{4}\right) \csc ^{2}\left(k x \mp \frac{\sqrt{2 k^{2}-\frac{3 i \sqrt{3}}{4}+\frac{3}{4}}}{\sqrt{2}} t\right)\right) \\
& u_{3,4}(x, t)=\ln \left(\frac{-1}{2}-i \frac{\sqrt{3}}{2}+\left(\frac{3}{4}+i \frac{\sqrt{3}}{4}\right) \csc ^{2}\left(k x \mp \frac{\sqrt{2 k^{2}+\frac{3 i \sqrt{3}}{4}+\frac{3}{4}}}{\sqrt{2}} t\right)\right) \\
& u_{5,6}(x, t)=\ln \left(-\frac{3}{2} \sec ^{2}\left(k x \mp \frac{1}{2} \sqrt{4 k^{2}-3} t\right)+1\right)
\end{aligned}
$$

\section{Conclusion}

Here, we applied the simple ansatz method under the Painlevé and traveling wave transformations, for solving the Tzitzéica type equations. We found the exact soliton and 
trigonometric function solutions of the TDBM and the TT equations through symbolic soft computation in Mathematica. Furthermore, these solutions might have significant importance in science and engineering fields. The study also showed that method is simple and easy to implement.

\section{Acknowledgement}

The research work of first author was supported by ORIC, CUI, Pakistan, under project NO: 16-85/CRGP/CIIT/VEH/1141.

\section{References}

[1] M Tzitzeica. Sur une nouvelle classe des surfaces. CR Acad. Sci. Paris, 150:955-956, 1910.

[2] Weiguo Rui. Exact traveling wave solutions for a nonlinear evolution equation of generalized tzitzéica-dodd-bullough-mikhailov type. Journal of Applied Mathematics, 2013(395628):1-14, 2013.

[3] Abdul-Majid Wazwaz. The tanh method: solitons and periodic solutions for the doddbullough-mikhailov and the tzitzeica-dodd-bullough equations. Chaos, Solitons and Fractals, 25:55-63, 2005.

[4] R. K. Dodd and R. K. Bollough. Polynomial conserved densities for the sine-gordon equations. Proc. R. Soc. Lond. A, 352:481-503, 1977.

[5] Robert Conte, Micheline Musette, and A. Michel Grunland. Backlund transformation of partial differential equations from the painleve-gambier classification ii. tzitzeica equation. Journal of Mathematical Physics, 40(4):2092-2106, 1999.

[6] Jun-Yi Zhu and Xian-Guo Geng. Darboux transformation for tzitzeica equation. Commun. Theor. Phys., 45:577-580, 2006.

[7] Alexander V. Mikhailov. The reduction problem and the inverse scattering method. Physica, 1(2):73-117, 1981.

[8] O. H. El-Kalaawy. Exact soliton solutions for some nonlinear partial differential equations. Chaos, Solitons and Fractals, 14:547-552, 2002.

[9] Kamruzzaman Khan and M. Ali Akbar. Exact and solitary wave solutions for the tzitzeica-dodd-bullough and the modified kdv-zakharov-kuznetsov equations using the modified simple equation method. Ain Shams Engineering Journal, 4:903-909, 2013. 
[10] Reza Abazari. The $\left(g^{\prime} / g\right)$-expansion method for tzitzéica type nonlinear evolution equations. Mathematical and Computer Modelling, 52(9-10):1834-1845, 2010.

[11] Jalil Manafian and Mehrdad Lakestani. Dispersive dark optical soliton with tzitzéica type nonlinear evolution equations arising in nonlinear optics. Optical and Quantum Electronics, 48(2):116, 2016.

[12] A. Korkmaz and K. Hosseini. Exact solutions of a nonlinear conformable time fractional parabolic equation with exponential nonlinearity using reliable methods. Optical and Quantum Electronics, 49(278):1-10, 2017.

[13] K Hosseini, Z Ayati, and R Ansari. New exact traveling wave solutions of the tzitzéica type equations using a novel exponential rational function method. Optik-International Journal for Light and Electron Optics, 148:85-89, 2017.

[14] Ahmed H Arnous, Aly R Seadawy, Rubayyi T Alqahtani, and Anjan Biswas. Optical solitons with complex ginzburg-landau equation by modified simple equation method. Optik-International Journal for Light and Electron Optics, 144:475-480, 2017.

[15] H. Rezazadeh, A. Korkmaz, M. Eslami, J. Vahidi, and R. Asghari. Traveling wave solution of conformable fractional generalized reaction duffing model by generalized projective riccati equation method. Optical and Quantum Electronics, 50(150):1-13, 2018.

[16] Q. Zhou, M. Mirzazadeh, E. Zerrad, A. Biswas, and B. Milivoj. Bright, dark, and singular solitons in optical fibers withspatio-temporal dispersion and spatially dependent coefficients. Jornal of Modern Optics., 63(10):427-430, 2016.

[17] Kamyar Hosseini, Peyman Mayeli, and Reza Ansari. Bright and singular soliton solutions of the conformable time-fractional klein-gordon equations with different nonlinearities. Waves in Random and Complex Media, 28(3):426-434, 2018.

[18] Alper Korkmaz. Exact solutions of space-time fractional ew and modified ew equations. Chaos, Solitons and Fractals, 96:132-138, 2017.

[19] AJAM Jawad, MD Petrovic, and A Biswas. Modified simple equation method for nonlinear evolution equations. Applied Mathematics and Computation, 217(2):869$877,2010$.

[20] B. Lu. The first integral method for some time fractional differential equations. $J$. Math. Anal. Appl., 395:684-693, 2012.

[21] M. Eslami. Trial solution technique to chiral nonlinear schrodinger's equation in $(1+2)-$ dimensions. Nonlinear Dynam, 85(2):813-816, 2016. 
[22] M. Younis and Asim Zafar. Exact solutions to nonlinear differential equations of fractional order via $\left(\frac{G^{\prime}}{G}\right)$-expansion method. Appl. Math, 5:1-6, 2014.

[23] Alper Korkmaz. Complex wave solutions to mathematical biology models i: Newellwhitehead-segel and zeldovich equations. Journal of Computational and Nonlinear Dynamics, 13(8):1-7, 2018.

[24] D. Kumar, K. Hosseini, and F. Samadani. The sine- gordon expansion method to look for the traveling wave solutions of the tzitzéica type equations in nonlinear optics. Optik - International Journal for Light and Electron Optics, 149:439 - 446, 2017.

[25] Alper Korkmaz. Exact solutions to $(3+1)$ conformable time fractional jimbo-miwa, zakharov-kuznetsov and modified zakharov-kuznetsov equations. Communications in Theoretical Physics, 67(5):479, 2017.

[26] Z. Ayati, K. Hosseini, and M. Mirzazadeh. Application of kudryashov and functional variable methods to the strain wave equation in microstructured solids. Nonlinear Engineering, 6:25-29, 2017.

[27] K. Hosseini, P. Mayeli, and D. Kumar. New exact solutions of the coupled sinegordon equations in nonlinear optics using the modified kudryashov method. Journal of Modern Optics, 65(3):361-364, 2018.

[28] K. Hosseini, F. Samadani, D. Kumar, and M. Faridi. New optical solitons of cubicquartic nonlinear schrödinger equation. Optik, 157:1101 - 1105, 2018.

[29] E. Fan. Extended tanh-function method and its application to nonlinear equation. Phys. Lett. A., 277:212-218, 2000.

[30] S. Zhang. Application of exp-function to a $\mathrm{kdv}$ equation with variable-coefficients. Phys. Lett. A, 365:448-453, 2007.

[31] AM. Wazwaz. The extended tanh method for the zakharov-kuznestsov(zk) equation, the modified zk equation, and its generalized forms. Commun. Nonlinear Sci., 13:10391047, 2008.

[32] Kamyar Hosseini, Jalil Manafian, Farzan Samadani, Mohammadreza Foroutan, Mohammad Mirzazadeh, and Qin Zhou. Resonant optical solitons with perturbation terms and fractional temporal evolution using improved $\tan (\phi(\eta) / 2)$-expansion method and exp function approach. Optik, 158:933 - 939, 2018.

[33] A. T. Ali and E. R. Hassan. General $\exp _{a}$ function method for nonlinear evolution equations. Appl. Math. Comput., 217:451-459, 2010. 
[34] K. Hosseini, Z. Ayati, and R. Ansari. New exact solution of the tzitzéica type equations in nonlinear optics using the $\exp _{a}$ function method. J. Mod. Opt., 65(7):847-851, 2018.

[35] E. M. E. Zayed and A. G. Al-Nowehy. Generalized kudryashov method and general $\exp _{a}$ function method for solving a high order nonlinear schrödinger equation. $J$. Space Explor., 6:1-26, 2017.

[36] Fuding Xie, Zhenya Yan, and Hongqing Zhang. Explicit and exact traveling wave solutions of whitham-broer-kaup shallow water equations. Physics Letters A, 285(1):76 $-80,2001$.

[37] Chenglin Bai. Exact solutions for nonlinear partial differential equation: a new approach. Physics Letters A, 288(3):191 - 195, 2001.

[38] K. Hosseini, A. Zabihi, F. Samadani, and R. Ansari. New explicit exact solutions of the unstable nonlinear schrödinger's equation using the $\exp _{a}$ and hyperbolic function methods. Optical and Quantum Electronics, 50(2):82, 2018.

[39] RI Nuruddeen. Multiple soliton solutions for the $(3+1)$ conformable space-time fractional modified korteweg-de-vries equations. Journal of Ocean Engineering and Science, 3(1):11-18, 2018. 